\title{
Absolute quantification of choline-related biomarkers in breast cancer biopsies by liquid chromatography electrospray ionization mass spectrometry
}

\author{
Maria Chiara Mimmi ${ }^{\mathrm{a}, *}$, Nicoletta Finato $^{\mathrm{b}}$, Gloria Pizzolato $^{\mathrm{a}}$, Carlo Alberto Beltrami ${ }^{\mathrm{b}}$, \\ Federico Fogolari ${ }^{\mathrm{a}}$, Alessandra Corazza ${ }^{\mathrm{a}}$ and Gennaro Esposito ${ }^{\mathrm{a}}$ \\ ${ }^{a}$ Department of Medical and Biological Sciences, University of Udine, Piazzale M. Kolbe, Udine, Italy \\ ${ }^{\mathrm{b}}$ Department of Pathology, University of Udine, Piazzale Santa Maria della Misericordia, Udine, Italy
}

\begin{abstract}
It has been repeatedly demonstrated that choline metabolism is altered in a wide variety of cancers. In breast tumours, the choline metabolite profile is characterized by an elevation of phosphocholine and total choline-compounds. This pattern is increasingly being exploited as biomarker in cancer diagnosis.

The majority of in vitro metabolomics studies, for biomarkers quantification in cell cultures or tissues, entail proton NMR spectroscopy. Although many "targeted" approaches have been proposed to quantify metabolites from standard one-dimensional (1D) NMR experiments, the task is often made difficult by the high degree of overlap characterizing ${ }^{1} \mathrm{H}$ NMR spectra of biological samples.

Here we present an optimized protocol for tissue extraction and absolute quantification of choline, phosphocholine and glycerophosphocholine by means of liquid chromatography electrospray ionization mass spectrometry (LC-ESI-MS). The selected chromatographic separation system with a HILIC (hydrophilic interaction chromatography) amide column effectively separates free choline and its phopshorylated derivatives, contrary to failure observed using standard reversed-phase chromatography. The metabolite absolute quantification is based on external calibration with commercial standards, and is validated by a parallel 1D proton NMR analysis.

The LC-MS/NMR analysis is applied to three breast carcinoma specimens obtained by surgical excision, each one accompanied by a control tissue sample taken outside the tumor margin. The metabolite concentrations measured are in good agreement with previous results on metabolic profile changes of breast cancer. Each of the three cancerous biopsies, when compared with the control tissue, exhibit a highly increased levels phosphocholine, total choline and phosphocholine/glycerophosphocholine ratio.
\end{abstract}

Keywords: Metabolomics, breast cancer, choline phospholipid metabolism, ${ }^{1} \mathrm{H}-\mathrm{NMR}$, LC-ESI-MS, hydrophilic interaction chromatography

\section{Background}

Choline (Cho) is a dietary component essential for the normal function of all cells [11]. The intracellular metabolism of choline in breast is partitioned among

*Corresponding author: Maria Chiara Mimmi, Department of Medical and Biological Sciences, University of Udine, Piazzale M. Kolbe 4, 33100 Udine, Italy. Tel.: +39 0432 494326; Fax: +39 0432 494301; E-mail: chiara.mimmi@uniud.it. two major pathways: (a) synthesis of phosphatidylcholine (PtdCho) and (b) oxidation to produce the methyl donor betaine.

Choline metabolism and choline-derived metabolites can exhibit remarkable alterations as a result of malignant proliferation. The abnormal choline metabolite profile typical of cancers is characterized by an elevation of phosphocholine (PCho) and total choline (tCho) containing metabolites. This has been 


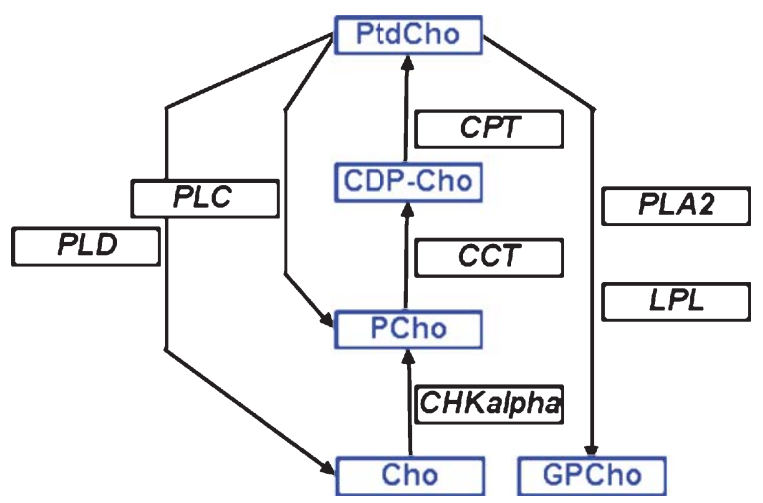

Fig. 1. Schematic diagram showing biosynthetic and catabolic pathways of phosphatidylcholine (PtdCho) metabolism. Metabolites are reported in blue with the following abbreviations: PtdCho, phosphatidylcholine; CDP-Cho, cytidine diphosphate choline; PCho, phosphocholine; Cho, choline; GPCho, glycerophosphocholine. Enzimes are reported in black: PLD, phospholipase D (EC:3.1.4.4); PLC, phospholipase C [EC:3.1.4.3]; CPT, choline phosphotransferase [EC:2.7.8.2]; CCT, choline-phosphate cytidylyltransferase [EC:2.7.7.15]; CHK-alpha, choline kinase alpha [EC:2.7.1.32]; PLA2, cytosolic phospholipase A2 [EC:3.1.1.4]; LPL, lysophospholipase I [EC:3.1.1.5].

demonstrated by numerous in vivo magnetic resonance spectroscopy (MRS) studies [4]. The major molecular causes of the increased PCho and tCho levels in cancer cells and tumors include an increased expression and activity of choline kinase alpha (CHKalpha), an increased activities of phosphatidylcholine-specific phospholipase C and D (PLC and PLD), and phospholipases A2 (PLA2) [5]. Moreover MRS investigation on breast cancers recognised the occurrence of a switch from high glycerophosphocholine (GPCho) and low PCho to low GPCho and high PCho in presence of cancerogenesis.

In synthesis all of the altered metabolites participate in the biosynthetic and catabolic pathways of the major membrane phospholipid PtdCho, as shown in the schematic diagram (Fig. 1).

In vivo detection of choline metabolites by proton magnetic resonance spectroscopy $\left({ }^{1} \mathrm{H}\right.$ MRS) has been proven to be useful in the diagnosis of cancer [10] and in monitoring the response of tumors to anticancer therapy [1]. However it is not possible to resolve free choline from its phosphorylated derivatives in vivo, even at high magnetic field strengths, because of the broad linewidths that result from restricted molecular motion and magnetic field inhomogeneities.

This is why the discovery of cancer biomarkers and potential drug targets necessarily involve metabolomic investigation on ex vivo samples by techniques such as high resolution NMR, mass spectrometry and high resolution magic angle spinning (HR-MAS).

The present work builds on the established correlation between choline metabolism modification and malignant tumor proliferation and focuses on three cancer indicators, namely tCho and PCho concentrations and PCho/GPCho ratio. A metabonomic study is conducted on three breast carcinoma specimens obtained by surgical excision, each one accompanied by a control tissue sample taken outside the tumor margin. The study entails tissue extraction of metabolites and a parallel analysis conducted by onedimensional (1D) ${ }^{1} \mathrm{H}$ NMR spectroscopy, taken as standard technique, and an optimized implementation of a LC/ESI-MS method.

The key feature of the method, which was already reported in 2011 [7], is the adoption of a polar stationary phase HILIC (the acronym stands for Hydrophilic Interaction LIquid Cromatography) that can retain polar compounds on the column without the disadvantages of using solvents that are immiscible with water, as used in traditional normal phase HPLC. The high amount polar organic mobile phase (acetonitrile) used in HILIC is especially compatible with ESI-MS, resulting in high sensitivity.

Scope of the present study is to prove that the assessment of breast cancer markers by LC/ESI-MS is feasible and diagnostically valuable. This technique shows a resolution advantage for assaying choline derivatives, and potentially many other target molecules, compared to NMR, and could complement the latter.

After the necessary large scale standardization of protocols, mass spectrometry (MS) could reduce the traumatic impact of current bioptic procedures based on its sensitivity and, eventually, replace the current diagnostic protocols. 
Table 1

Specimens features summary

\begin{tabular}{|c|c|c|c|c|}
\hline Patient & Specimen & Histology & Degree & Specimen weight \\
\hline \multirow[t]{2}{*}{ A } & 1 & Healthy tissue control & & $0,1668 g$ \\
\hline & 2 & Infiltrating lobular carcinoma & II & $0,2334 g$ \\
\hline \multirow[t]{2}{*}{ B } & 3 & Healthy tissue control & & $0,3127 \mathrm{~g}$ \\
\hline & 4 & Infiltrating ductal carcinoma & II & $0,2783 g$ \\
\hline \multirow[t]{2}{*}{$\mathrm{C}$} & 5 & Healthy tissue control & & $0,1301 \mathrm{~g}$ \\
\hline & 6 & Infiltrating ductal carcinoma & III & $0,1243 g$ \\
\hline
\end{tabular}

\section{Experimental section}

\subsection{Reagents}

Standard compounds were obtained from SigmaAldrich. Choline, phosphocholine and glycerophosphocholine were respectively in the form of choline chloride, phosphorylcholine chloride calcium salt tetrahydrate and sn-glycero-3-phosphorylcholine 1:1 cadmium chloride adduct. All solvents used were of HPLC grade.

\subsection{Instrumentation}

The LC/MS system consisted of a QqTOF mass spectrometer (Q-STAR by Applied BiosystemsSCIEX) equipped by an electrospray ionization (ESI) source and an Agilent 1100 series micro LC pump.

${ }^{1} \mathrm{H}$ NMR experiments were acquired on a Bruker Avance spectrometer operating at $500 \mathrm{MHz}$.

\subsection{Tissue extraction}

Human breast-tissue specimens were obtained from three patients (referred to as patients A, B, C) by surgical excision (Table 1). In any case a double resection was performed, the first from the active proliferating carcinoma and the second from healthy tissue by sampling $2 \mathrm{~cm}$ away from the tumor margin. The samples were immediately frozen in liquid nitrogen after collection and then stored at $-80^{\circ} \mathrm{C}$ until the time of metabolites extraction and analysis.

Choline compounds were extracted from tissue by a method derived from Bligh and Dyer [3,6]. The frozen samples were pulverized by an ULTRA-TURRAX T25 Homogenizer (IKA). To prevent thawing the biopsies were put in plastic test tube and kept dipped into liquid nitrogen during the whole smashing process. The frozen tissue from mammary specimens was weighted after pulverization and ranged roughly between 100 and $300 \mathrm{mg}$. Immediately after weighting, the following extraction protocol was performed (solvents volumes are related to $100 \mathrm{mg}$ of pulverized tissue):

1) The frozen powder was suspended in $0.400 \mathrm{ml}$ methanol/chloroform $(2: 1 \mathrm{v} / \mathrm{v})$ and, after thorough vortexing, left overnight at $-20^{\circ} \mathrm{C}$.

2) The sample was then centrifuged at $1500 \mathrm{~g}$ for 5 minutes and the supernatant extract was separated and stored at $-20^{\circ} \mathrm{C}$.

3) The residue was submitted to re-extraction with $0.250 \mathrm{ml}$ of methanol/chloroform/water $(2: 1: 0.8$ by volume) and left at $-20^{\circ} \mathrm{C}$ for at least four hours.

4) The supernatants from both extractions were combined and, after addition of first $0.100 \mathrm{ml}$ of chloroform and then $0.100 \mathrm{ml}$ of water, the resulting solution was submitted to 5 minutes centrifugation. The procedure separates two phases: an aqueous phase containing methanol at the top and an organic phase at the bottom; in some case denatured proteins was observed in the middle.

5) The aqueous phase (that contains the hydrosoluble choline compounds) was directly lyophylized and then redissolved in $0.020 \mathrm{ml}$ water and $0.800 \mathrm{ml}$ methanol $\left(2.4 \% \mathrm{H}_{2} \mathrm{O}\right.$ by volume $)$. The resulting minute amounts of precipitates (proteins) were removed by filtration with $0.2 \mu \mathrm{m}$ filters. The filtrated solution was stored at $-80^{\circ} \mathrm{C}$ until it was used for either LC/ESI-MS or NMR analysis.

\section{4. $L C / E S I-M S$}

\subsubsection{Analysis}

The LC-MS method previously reported [7] was adopted with minor modifications. Aqueous extracts from mammary tissue samples were dried and rediluted 
in a mixture of of acetonitrile/water/methanol $=7 / 1 / 2$ and then submitted to LC separation and MS analysis (injected volume $5 \mu \mathrm{l}$, corresponding to the content of $1 \mathrm{mg}$ of pulverized tissue). Chromatographic separation of choline compounds, namely choline (Cho), phosphorylcholine (PCho) and glycerophosphorylcholine (GPCho) was obtained with a HILIC TSK-Gel Amide-80 column $5 \mu \mathrm{m}$ (length $150 \mathrm{~mm}$ and ID $0.50 \mathrm{~mm}$ from GRACE). The eluent composition was: $\mathrm{H}_{2} \mathrm{O} 99.9 \%, 0.1 \% \mathrm{HCOOH}$ and $5 \mathrm{mM}$ $\left[\left(\mathrm{NH}_{4}\right)^{+}\left(\mathrm{CH}_{3} \mathrm{COO}\right)^{-}\right]$for mixture $\mathrm{A} ; 97.9 \% \mathrm{CH}_{3} \mathrm{CN}$, $2.0 \% \mathrm{H}_{2} \mathrm{O}$ and $0.1 \% \mathrm{HCOOH}$ for mixture $\mathrm{B}$. The following 60-minute gradient program was used: from 0 th to 5 th minute column conditioning at $80 \%$ of $\mathrm{B}$; from 5 th to 40 th minute, B gradient from $80 \%$ to $10 \%$; from 40th to 45 th minute, B percentage fixed at $80 \%$; finally from 45 th to 60 th minute back-gradient from $10 \%$ to $80 \%$ of mixture B. The flow rate was set at $10 \mu \mathrm{L} / \mathrm{min}$.

Positive ESI parameters for standard MS scan were tuned for best sensitivity under HPLC conditions. Nitrogen was used as nebulizer gas (GS1) and curtain gas (CUR): GS1 and CUR were set respectively at 20.00 and 15.00 psi. The DP (declustering potential), FP (focusing potential) and DP2 (secondary declustering potential) were set respectively at 40, 230 and $15 \mathrm{~V}$. The ion spray voltage (IS) was $5 \mathrm{kV}$. The positively charged molecular ions of choline compounds were monitored by extracting the ion chromatogram of selected mass ranges including the target molecules mass.

\subsubsection{Data treatment}

The analytes peaks were integrated with the software Analyst QS 1.1 (Applied Biosystems-SCIEX) by evaluating the extracted ion content (XIC) counts. The correspondence between measured XIC counts and absolute concentrations was established by calibration curves with standard compounds.

Details can be found in the Supplementary Material Section.

\subsection{NMR spectroscopy}

\subsubsection{Analysis}

An aliquot of each aqueous extract (corresponding to $75 \mathrm{mg}$ of frozen pulverized tissue) was dried under vacuum and redissolved in $0.5 \mathrm{~mL} \mathrm{D}_{2} \mathrm{O}$ with the addition of $2 \mathrm{~mL}$ of $\mathrm{D}_{2} \mathrm{O}$ with $0.075 \%$ of sodium 3-trimethyl-silyl [2,2-3,3- $\left.d_{4}\right]$ propionate (TSP) in $\mathrm{D}_{2} \mathrm{O}$ as a frequency reference. The working temperature was $298 \mathrm{~K}$.

Monodimensional ${ }^{1} \mathrm{H}$ spectra were collected using a sweep width of $8 \mathrm{kHz}$ and a time domain of $8 \mathrm{~K}$ complex data points, with 64 acquisitions and 8 silent scans. Flip-angle pulses of $30^{\circ}$ were applied with a relaxation delay of $5 \mathrm{~s}$. Presaturation was used for suppression of the HOD signal during the whole initial delay. The adopted pulse sequence includes the Electronic Reference Signal (ERETIC ${ }^{\mathrm{TM}}$ ) [2] synthesized by an electronic device, which was used for the determination of absolute concentrations. The reference signal provides a pseudo-FID that has all the characteristics of a real NMR signal, after proper calibration, and whose parameters (frequency, magnitude, phase, T2) are controlled from the spectrometer console. Spectra acquisition and processing were performed with the Bruker software TOPSPIN.

\subsubsection{Data treatment}

After FID apodization with a shifted sine square window function $\left(90^{\circ}\right)$ all spectra were phased, Fourier-transformed and chemical-shift referenced. The integral value of significant peaks were measured. In some cases Gaussian window multiplication of the FID was necessary to enhance resolution of signals: the deconvolution of the envelopes of interest was performed by fitting a mixed Lorentzian/Gaussian lineshape to the experimental pattern. The peak modeling,<smiles>C[N+](C)(C)CCO</smiles><smiles>C[N+](C)(C)CCOP(=O)(O)O</smiles>

Phosphocholine<smiles>C[N+](C)(C)CCOP(=O)(O)OC[C@H](O)CO</smiles>

Fig. 2. Chemical structure of Choline (Cho), Phosphocholine (PCho) and sn-Glycero-3-phosphocholine (GPCho). 
performed with TOPSPIN, was used only for the estimate of the integral fractions to be assigned to the single components of peak envelopes.

The absolute quantification of metabolites of interest (namely Cho, PCho, GPCho) was based on N-trimethyl peaks (Fig. 2), which are the most intense and isolated in mono-dimensional spectra, relative to the ERETIC signal. The artificial peak was used as an internal standard whose equivalent concentration [ERETIC] was calibrated against the absolute concentrations of three standard compounds. Further details can be found in the Supplementary Material Section.

\section{Results}

\subsection{Extraction protocol}

The very first step of frozen tissue pulverization was modified with respect to our previously reported protocol [7]. This is crucial for samples as inhomogeneous as typical mammary biopsies. In particular instead of grinding the frozen sample with a scalpel, we used a homogenizer (model ULTRA-TURRAX T25, by IKA) provided with a stainless steel disperser tool. The small diameter of the steel tip $(8 \mathrm{~mm})$ allowed working on biopsies which were located in plastic test tube and kept dipped into liquid nitrogen during the whole smashing process. This method resulted in an efficient grinding of tissue: the whole operation could be accomplished in 5-10 minutes depending on the sample. Furthermore keeping biopsies dipped into liquid nitrogen avoids sample thawing, a bad eventuality for chemical stability of labile metabolite and for their reliable quantification.

\subsection{LC/ESI-MS}

The LC/ESI-MS analysis of the bioptic aqueous extracts focused on three significant metabolites for cancer diagnosis: Cho, PCho, and GPCho. The three molecules are hydrosoluble and positively charged, due to the choline moiety, hence all are detected by ESI-MS under positive mode.

The protocol for chromatographic separation and mass spectrum acquisition had been already established [7], therefore only small modifications were made, to improve the chromatographic separation (see Experimental Section).
The Total Ion Current (TIC) Chromatogram of a standard mixture solution of Cho, PCho, GPCho is shown in Fig. 3; the HILIC separation efficiency for the Cho compounds can be appreciated by looking at the extracted ion chromatograms (XICs) obtained by selecting the specific $\mathrm{m} / \mathrm{z}$ values and thus discarding the baseline and co-eluting ions. The quantification of Cho compounds in the biological samples was based on their XICs: representative samples chromatograms are shown in Fig. 4.

The concentration of free Cho and Cho derivatives obtained by LC/ESI-MS analysis are reported in Table 2.

\section{3. $N M R$}

Bioptic aqueous extracts, obtained as described in Experimental Section, were analyzed by ${ }^{1} \mathrm{H} 1 \mathrm{D}$ NMR without any buffer addition. Examples of full spectra relative to two representative biologic samples are shown in Fig. 5; the enlarged choline region of the same spectra is reported in Fig. 6 and compared to a mixture of standard Cho, PCho and GPCho in $\mathrm{D}_{2} \mathrm{O}$. The quantification of Cho, PCho and GPCho exploited the resonances of the respective $\mathrm{N}$-trimethyl groups that originate very intense singlets. The specific peaks were identified in the region between 3,15 and 3,35 ppm where, besides choline and derivatives, a number of other signals occur (phosphoethanolamine, taurine, betaine, etc.). The chemical shifts of the Ntrimethyl groups vary depending on the sample $\mathrm{pH}$; in neutral solutions the chemical shifts are: $3,20 \mathrm{ppm}$ for Cho, 3,22 ppm for PCho and 3,23 ppm for GPCho. The assignment was based on a report by Sitter et al. [9] and was confirmed by comparison with the spectra of synthetic standard mixtures recorded at different $\mathrm{pH}$ conditions (data not reported).

The Cho compounds estimation resulting by 1D NMR analysis is reported in Table 3.

\section{Discussion}

\subsection{Sample choice}

The first qualifying point of the present study is the availability of a double specimen for each of the three enrolled patients: the first derived from the proliferating carcinoma and the second from healthy tissue 


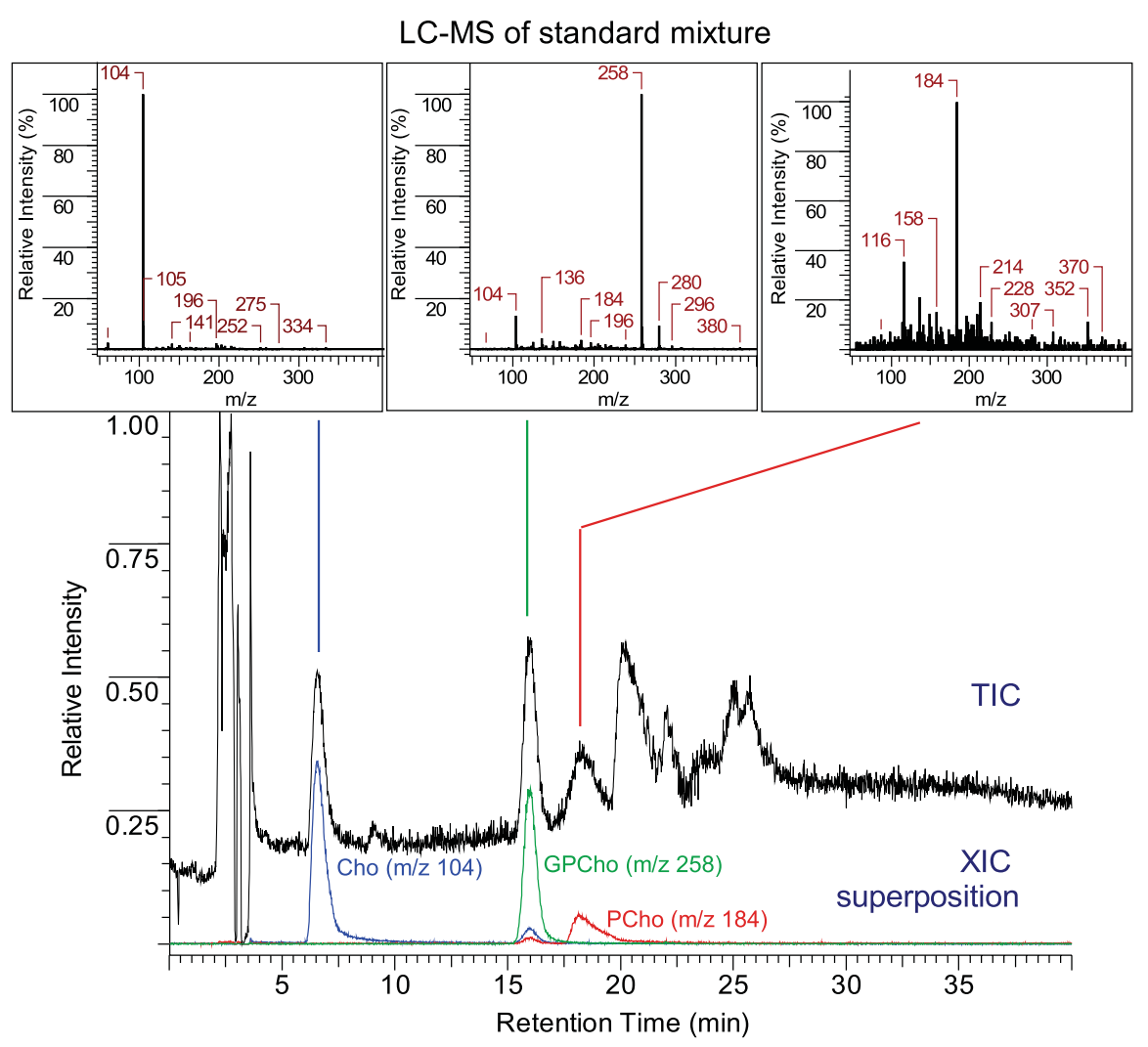

Fig. 3. LC/ESI-MS analysis of a standard mixture solution of Cho, PCho, GPCho in acetonitrile/water $/ \mathrm{methanol}=7 / 1 / 2=\mathrm{v} / \mathrm{v} / \mathrm{v}$. Together with the Total Ion Current (TIC) Chromatogram (black trace), the extracted ion chromatogram (XIC) for Cho (blue trace), PCho (red traces), GPCho (green trace) are reported. The XIC chromatograms are obtained by selecting the suitable m/z values (namely 104 for free Cho, 184 for PCho and 258 for GPCho) and provide the elution curves of the three compounds. The three top panels represent the TOF-MS spectra acquired at elution of the three peaks. The Cho and PCho components occurring at the retention time of GPCho (at ca 16 min) are due to in-source fragmentation of the latter metabolite.

outside the tumor margin. Though in principle every metabonomic study should consider cancer biopsies and healthy tissue controls from the same individuals, i.e. internal controls, to account for the individual metabolome variability, this is not always possible and many theoretical conclusions on metabolic markers are based on the differential absolute concentrations in cancerous and normal tissues, without taking into account the tissue source. In this study, on the contrary, we could exclude that the concentration changes of choline compounds were related to the intrinsic variability among individuals.

\subsection{Methodology: NMR vs LC-MS}

NMR and Mass Spectrometry (MS) represent the principal approaches in metabolomics/metabonomics studies. In this type of application, NMR has many advantages such as high information content of the resulting spectra, ease of quantification and little need of optimization for the analysis conditions. MS techniques can cover a wide chemical diversity with high sensitivity and resolution, but require a preliminary optimization of the chromatographic separation.

The present study highlights the complementarity of the two analytical approaches. We adopted 1D NMR as conventional reference technique to collect a full set of information on the aqueous extract of biopsies. The use of 1D NMR for the analysis of unfractionated tissue extract, with hundreds of overlapping resonances can not afford complete assignment and accurate peak integration. For the quantitative determination of our target biomarkers with well established assignment, however, we could adopt the approach of fitting overlapped 1D spectra with modeled peaks. The modeling 

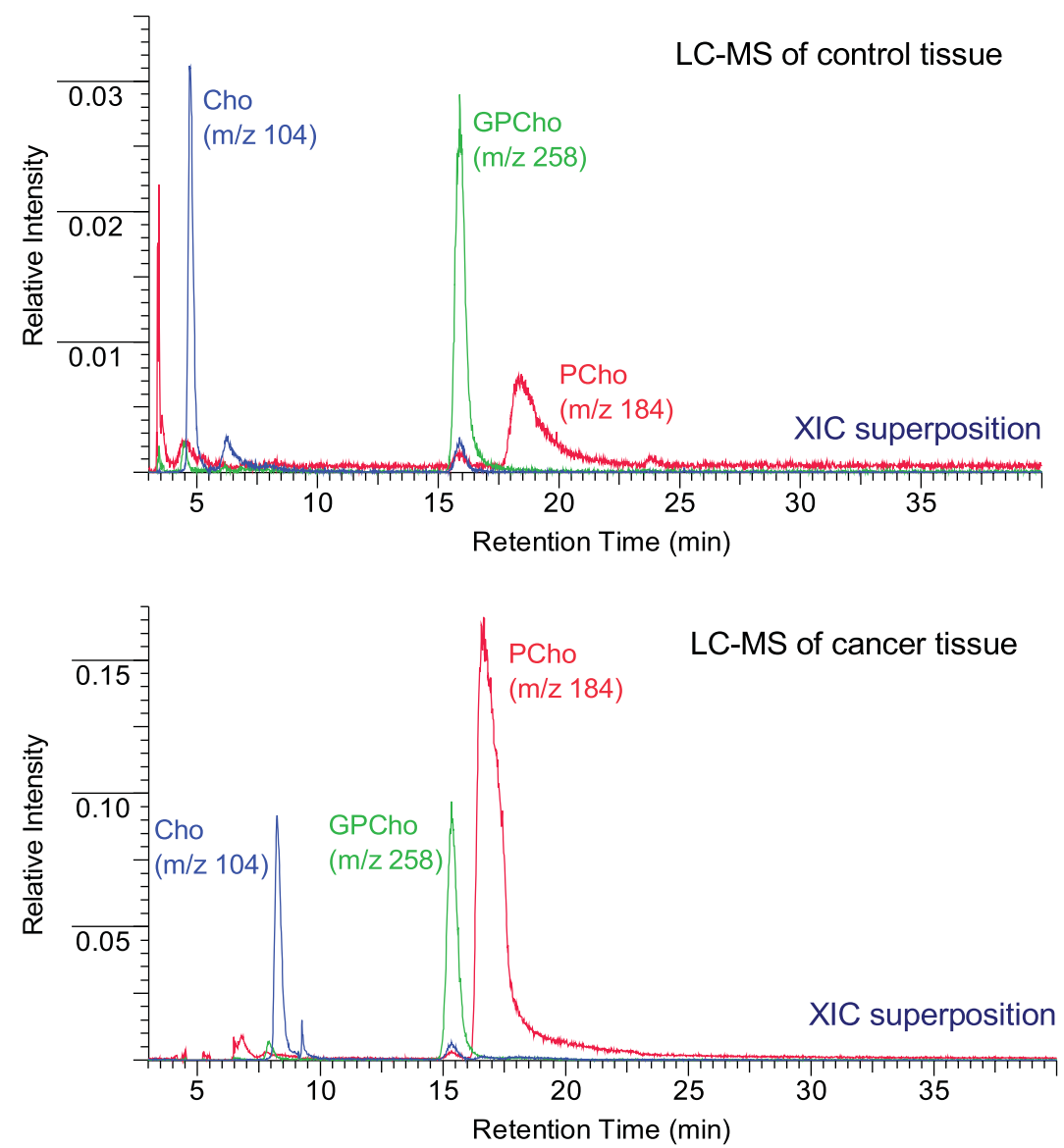

Fig. 4. Examples of LC/ESI-MS analysis of aqueous extracts derived from healthy breast tissue (top panel, sample C-5) and cancerous breast tissue (bottom panel, sample C-6). Only the extracted ion chromatograms (XICs) are shown for Cho (blue traces), PCho (red traces), GPCho (green traces). The Cho and PCho components occurring at the retention time of GPCho (at 15-16 min) are due to in-source fragmentation of the latter metabolite. The splitting of the Cho peak into two peaks is likely to be ascribed to the different possible mechanisms of interactions of the molecule with the HILIC solid phase at the operating conditions. A remarkable difference in the relative intensity of Cho, PCho and GPCho signals is found and is consistent with cancer-related metabolism alteration.

approach allowed the concentration estimate of Cho, PCho and GPCho in the whole set of sample, though for 2 out of 6 samples it was not possible to resolve PCho and GPCho, and only the total amount of the two compounds was reported.

Differently, the combination of on-line HILIC separation and ESI-MS allowed to resolve the target peaks without the necessity of chemical derivatization. The LC-MS quantification of Cho derivatives in the test samples was obtained by interpolation of the LC-MS response, based on calibration curves obtained under the same experimental conditions. Although LC-MS quantitative assessment is safely carried out based on isotopically labeled internal standards, the response for
Cho compounds is reasonably linear over the working range (correlation coefficients between 0.9686 and 0.9892 low $\mathrm{R}^{2}$ values due to random errors). Some critical aspects of the LC-MS approach, that arise from reproducibility of the measurements, remain inherently unavoidable. Some significant metabolite level differences observed in Tables 2 and 3 could be due to the inherent reproducibility differences of the LCMS and NMR techniques, as well as to plausible differences in hydrolysis pattern of the Cho metabolite pools in the samples submitted to either LC-MS or NMR analysis. Further analysis of sample groups of significant size will more properly address this issue. 
Table 2

Results of LC-MS estimate of Cho and its phosphorylated derivatives concentrations. The confidence limits were derived from equation (1) of supplementary material section

\begin{tabular}{lcccc}
\hline Patient & Specimen & $\begin{array}{c}\text { Choline } \\
\text { pmol/mg }\end{array}$ & $\begin{array}{c}\text { Phosphocholine } \\
\text { pmol/mg }\end{array}$ & $\begin{array}{c}\text { Glycerophosphocholine } \\
\text { pmol/mg }\end{array}$ \\
\hline A & 1 & $25 \pm 24$ & $215 \pm 115$ & $43 \pm 23$ \\
& 2 & $141 \pm 24$ & $1281 \pm 115$ & $70 \pm 23$ \\
B & 3 & $128 \pm 12$ & $56 \pm 58$ & $35 \pm 12$ \\
& 4 & $17 \pm 12$ & $605 \pm 58$ & $145 \pm 12$ \\
C & 5 & $5 \pm 3$ & $62 \pm 58$ & $28 \pm 12$ \\
& 6 & $46 \pm 12$ & $779 \pm 58$ & $115 \pm 12$ \\
\hline
\end{tabular}
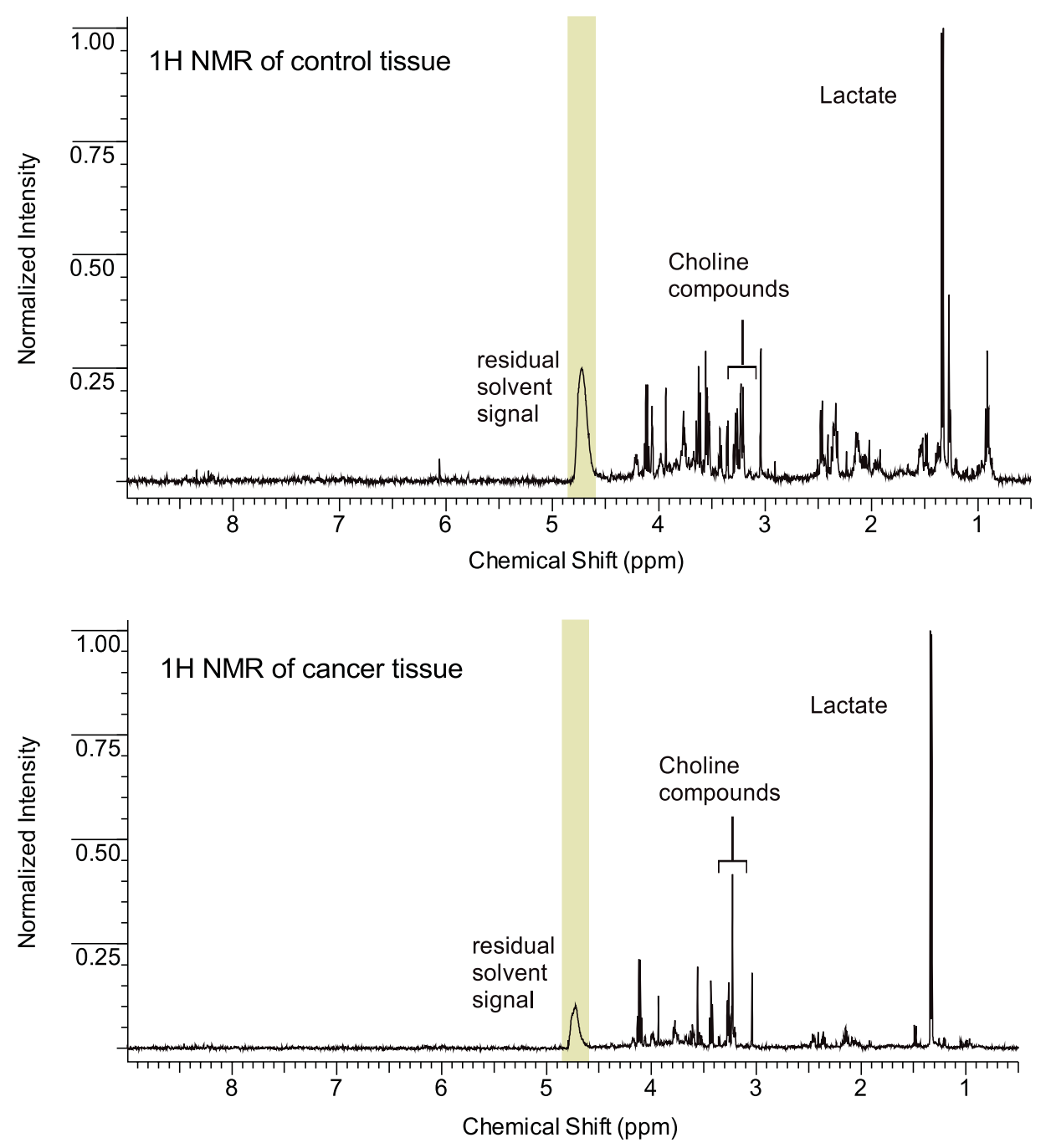

Fig. 5. Examples of $500 \mathrm{MHz}{ }^{1} \mathrm{H}$ NMR spectra obtained from biopsy aqueous extracts. The top panel refers to healthy breast tissue (sample A-1) and the bottom panel refers to cancerous breast tissue (sample A-2). The region of the $N$-trimethyl groups of choline compounds and the peak of lactate (the most intense resonance in both spectra) are indicated. The residual solvent signal is highlighted. 
Table 3

Results of 1D NMR estimate of Cho and its phosphorylated derivatives concentrations. The errors were derived from equation (4) (Supplementary Material Section). For specimens 3 and 5 the deconvolution of PCho and GPCho peaks was not possible, therefore only the total concentration estimate is reported

\begin{tabular}{lccccc}
\hline Patient & Specimen & $\begin{array}{c}\text { Choline } \\
\text { pmol/mg }\end{array}$ & $\begin{array}{c}\text { Phosphocholine } \\
\text { pmol/mg }\end{array}$ & $\begin{array}{c}\text { Glycerophosphocholine } \\
\text { pmol/mg }\end{array}$ & $\begin{array}{c}\text { Phosphocholine + Glycerophosphocholine } \\
\text { pmol/mg }\end{array}$ \\
\hline A & 1 & $113 \pm 9$ & $101 \pm 8$ & $76 \pm 6$ & $178 \pm 10$ \\
& 2 & $120 \pm 9$ & $966 \pm 72$ & $67 \pm 5$ & $1032 \pm 72$ \\
B & 3 & $9 \pm 1$ & nd & nd & $41 \pm 3$ \\
& 4 & $81 \pm 6$ & $589 \pm 44$ & nd & $67 \pm \pm 44$ \\
C & 5 & $24 \pm 2$ & $634 \pm 47$ & $143 \pm 11$ & $107 \pm 8$ \\
& 6 & $107 \pm 8$ & & & $777 \pm 48$ \\
\hline
\end{tabular}

\subsection{Breast cancer diagnosis: Implications of choline-containing biomarkers}

As reviewed by Podo [8] in 1999 and by Glunde et al. in 2006 [4], elevated PCho content represents a general feature of most tumours and is related to the enhancement of the so-called ${ }^{1} \mathrm{H}$ "choline peak" (tCho) centered at $3.2 \mathrm{ppm}$ and observed in MR spectra of cancerous tissue in vivo. [the tCho peak may actually comprise signals from choline-containing compounds, such as GPCho and PCho, as well as free Cho itself, together with contributions from other metabolites, such as phosphoethanolamine, inositol and taurine]. It should be considered that PCho alteration is not exclusive to malignancy, since it may also occur in other cases of accelerated tissue proliferation like for instance benign tumours or developing (embryo or fetal) organs.

According to Glunde, in breast cancers, a switch from high GPCho and low PCho to low GPCho and high PCho is precisely correlated with malignant transformation of cells. In addition several lines of evidence confirm the significance of the PCho/GPCho ratio as a possible indicator of altered phospholipid turnover during tumour progression; while elevated concentrations of PCho may simply reflect high rate of cell proliferation (for either tumour or normal cells). Consequently, the role of specific indicator of malignancy might be attributed to the PCho/GPCho ratio. These concepts were the starting point of the present study which aimed at establishing robust analytical methods for measuring choline related cancer biomarkers such as PCho level, PCho/GPCho ratio, and tCho level in tissue samples. Indeed our results demonstrate that even significant metabolite level differences estimated by different techniques are compensated when analysed within the interpretation frame above defined
(Figs. 7 and 8). Therefore we consider our results a proof of concept and propose the collection of a statistically representative data pool, to improve the definition of quantitative standards. Based on the current LCMS and NMR analysis, the $\mathrm{PCho}_{\text {tumour }} / \mathrm{PCho}_{\text {control }}$ ratio varies between 6 and 13, while the indicator PCho/GPCho increases by some 3 to 7 -fold in cancer lesion compared to healthy tissue.

\section{Concluding remarks}

The extraction and analytical methods described in this study offer useful tools to determine absolute metabolite concentrations in tissue extracts, and more generally in complex biological mixtures. An optimized implementation of a LC/ESI-MS method for the absolute determination of Cho and its phosphorylated derivatives is compared with a $1 \mathrm{D}-{ }^{1} \mathrm{H}$ NMR spectroscopy method entailing an internally-synthesized radiofrequency reference such as ERETIC ${ }^{\mathrm{TM}}$.

Besides possible sample alterations, we encountered limited reproducibility problems in LC/ESI-MS quantifications related to external calibrations that can be avoided using an internal isotopically-labelled reference standard.

In addition the complexity of tissue extracts metabolomics is well known, especially when compared to biofluids or cell lines studies, because of the heterogeneity of tissues resections undergoing pulverization and extraction and the high dynamic range among metabolite concentrations.

In spite of these drawbacks, a reasonable agreement between LC/ESI-MS and NMR analysis was found and an efficient discrimination between control and cancerous tissue was possible. 

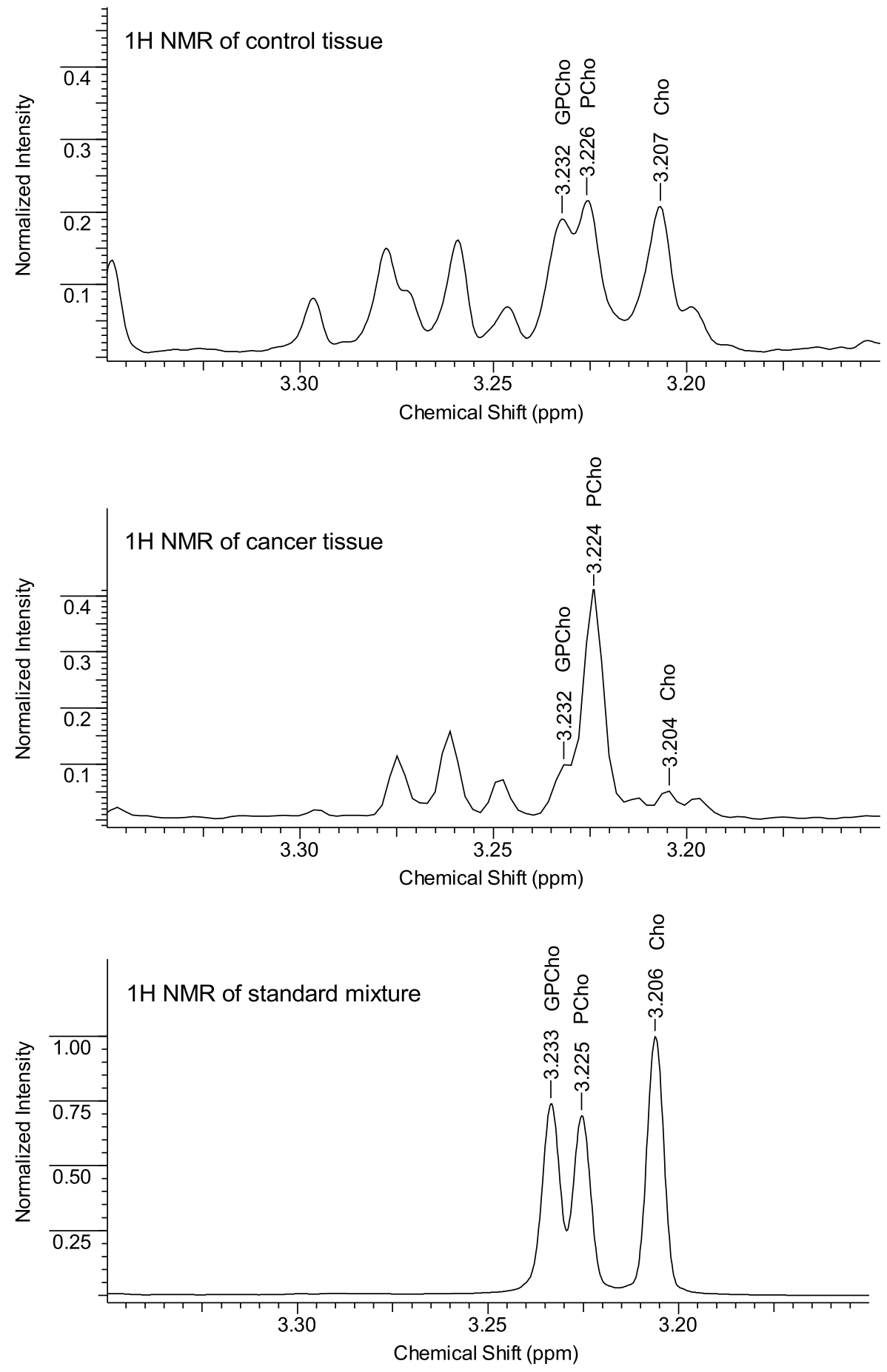

Fig. 6. Selected region from $500 \mathrm{MHz}{ }^{1} \mathrm{H}$ NMR spectra of biopsy aqueous extracts. The top and middle panel refer to healthy and cancerous breast tissue, respectively sample A-1 and sample A-2. The bottom panel refer to a mixture of standard Cho, PCho and GPCho in $\mathrm{D}_{2} \mathrm{O}$. The measured $\mathrm{pH}$ of the three samples varied in the range 6,8-7,2. The $N$-trimethyl peaks of Cho and derivatives are labeled with the corresponding chemical shifts. The remarkable intensity differences are consistent with cancer-related metabolism modification. 


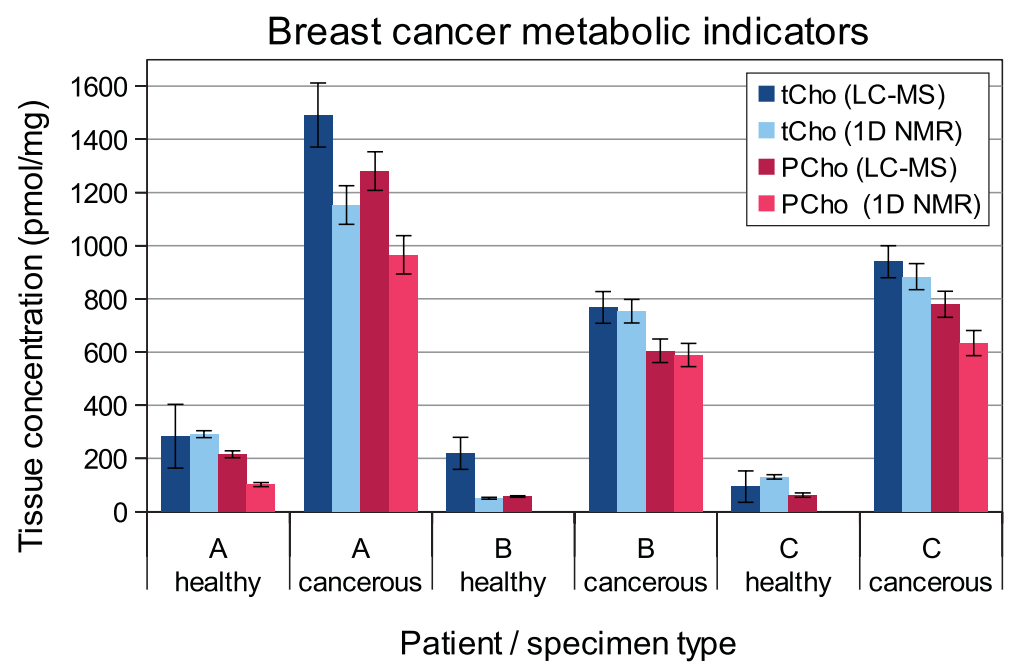

Fig. 7. Bar chart representation of tissue concentration of: total Choline compounds (tCho) and phosphocholine (PCho) in bioptic aqueous extracts.

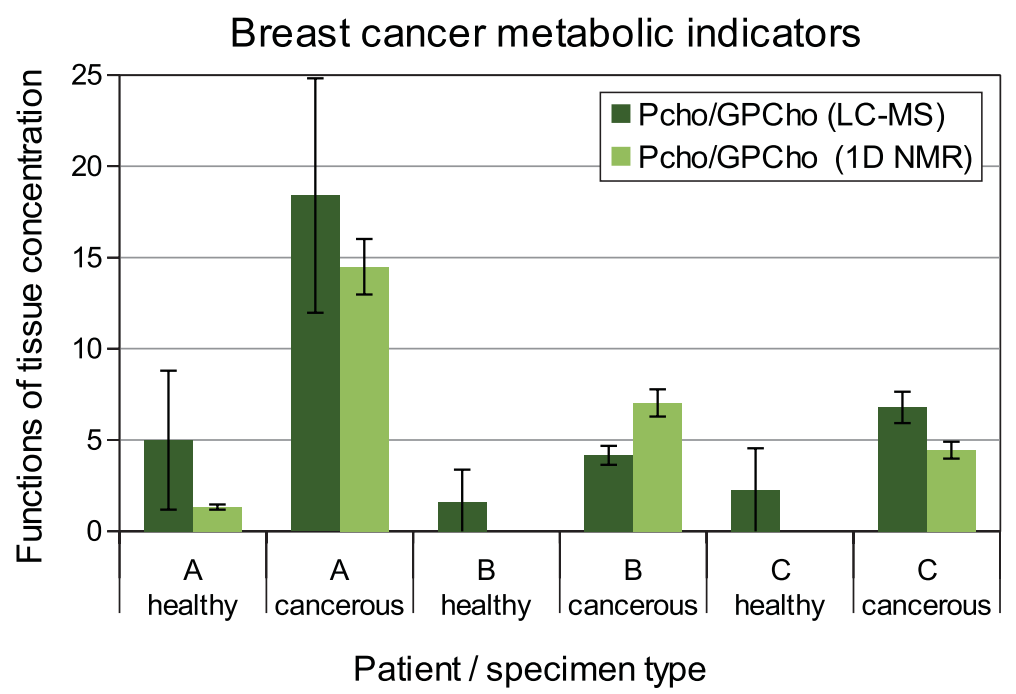

Fig. 8. Bar chart representation of the ratio of PCho and GPCho tissue concentrations in bioptic aqueous extracts. Both LC-MS and NMR results are shown, where available.

While the three different biopsies, with the relative healthy tissue counterparts, here analyzed represent a proof of concept of significance and robustness of tCho and PCho levels and PCho/GPCho ratio, the whole protocol will need to be extended to a significant number of samples in order to validate statistically a sound quantitative refinement of its potentialities.

Once a proper statistical size will be available, the data set collected by NMR and LC/ESI-MS could be analyzed from the perspective of untargeted metabolomics as well. In fact it should be possible to apply multivariate statistics in order to verify the possibility of new correlation between metabolic alterations and malignant proliferation.

With respect to more conventional diagnostic tools, such as histology or immunohistochemistry, NMR and MS provide a large amount of information that enable database processing to extract unique correlation. 


\section{Acknowledgments}

This work was financially supported by the Italian Ministry for University and Research (FIRB RBRN07BMCT) and European Union (LSHM-CT2005-037525).

\section{References}

[1] S. Akoka, L. Barantin and M. Trierweiler, Concentration Measurement by Proton NMR Using the ERETIC Method. Anal Chem 71(13) (1999), 2554-2557.

[2] J.N. Miller and J.C. Miller, Statistics and Chemometrics for Analytical Chemistry. Pearson Education Limited, 6th ed. 2010. Chapter 5.6: 121-122.

[3] H.M. Baek, J.H. Chen, K. Nie, H.J. Yu, S. Bahri, R.S. Mehta, O. Nalcioglu and M.Y. Su, Predicting pathologic response to neoadjuvant chemotherapy in breast cancer by using MR imaging and quantitative 1H MR spectroscopy, Radiology 251 (2009), 653-662.

[4] L. Barantin, A. Le Pape and S. Akoka, A new method for absolute quantitation MRS metabolites. Magn Reson Med 38 (1997), 179-182.

[5] E.G. Bligh and W.J. Dyer, A rapid method of total lipid extraction and purification, Can J Biochem Physiol 37(8) (1959), 911-917.

[6] K. Glunde, M.A. Jacobs and Z.M. Bhujwalla, Choline metabolism in cancer: Implications for diagnosis and therapy, Expert Review of Molecular Diagnostics 6(6) (2006), 821829.

[7] K. Glunde, L. Jiang, S.A. Moestue and I.S. Gribbestad, MRS and MRSI guidance in molecular medicine: Targeting and monitoring of choline and glucose metabolism in cancer, $N M R$ Biomed 24 (2011), 673-690.

[8] H. Koc, M.H. Mar, A. Ranasinghe, J.A. Swenberg and S.H. Zeisel, Quantitation of choline and its metabolites in tissues and foods by liquid chromatography/electrospray ionizationisotope dilution mass spectrometry, Anal Chem 74 (2002), 4734-4740.

[9] M.C. Mimmi, P. Picotti, A. Corazza, E. Betto, C.E. Pucillo, L. Cesaratto, C. Cedolini, V. Londero, C. Zuiani, M. Bazzocchi and G. Esposito, High-performance metabolic marker assessment in breast cancer tissue by mass spectrometry, Clin Chem Lab Med 49(2) (2011), 317-324.

[10] F. Podo, Tumor Phospholipid metabolism, NMR in Biomedicine 12 (1999), 413-439.

[11] B. Sitter, U. Sonnewald, M. Spraul, H.E. Fjosne and I.S. Gribbestad, High-resolution magic angle spinning MRS of breast cancer tissue, NMR Biomed 15 (2002), 327337.

[12] P. Stanwell, L. Gluch, D. Clark, B. Tomanek, L. Baker, B. Giuffre, C. Lean, P. Malycha and C. Mountford, Specificity of choline metabolites for in vivo diagnosis of breast cancer using ${ }^{1} \mathrm{H}$ MRS at 1.5 T, Eur Radiol 15 (2005), 1037-1043.

[13] S.H. Zeisel, Choline: Critical role during fetal development and dietary requirements in adults, Annu Rev Nutr 26 (2006), 229-250.

\section{Supplementary material}

\section{LC/ESI-MS}

\section{Calibration curve}

Three stock solutions $1 \mathrm{mM}$ of Cho, PCho and GPCho were prepared in acetonitrile/water $=1 / 1$.

Several diluent mixture containing variable percentage of acetonitrile/water/methanol were tested for the LC-MS analysis and the best compromise for solubility, peak shape and resolution resulted to be a mixture of acetonitrile/water/methanol $=7 / 1 / 2$.

Six solutions containing the three standards at increasing concentrations, selected to include biological sample metabolites levels, were injected in the LC-MS system for calibration. The concentration range and regression results for each standard is reported in Table 4.

\section{Data treatment}

The same diluent mixture and the same set of experimental conditions was adopted for calibration curve building and for samples measurements. For each determined concentration, $\mathrm{x}_{0}$, the random error, $\mathrm{s}_{\mathrm{x} 0}$, was estimated from calibration curves using the formula (1):

$$
\mathrm{s}_{x o}=\frac{\mathrm{s}_{\mathrm{y} / \mathrm{x}}}{\mathrm{b}}\left\{\frac{1}{\mathrm{~m}}+\frac{1}{\mathrm{n}}+\frac{\left(\mathrm{y}_{0}-\mathrm{y}_{a v}\right)^{2}}{\mathrm{~b}^{2} \sum_{\mathrm{i}}\left(\mathrm{x}_{\mathrm{i}}-\mathrm{x}_{a v}\right)^{2}}\right\}^{1 / 2}
$$

$s_{y / x}$ is the standard deviation of the ordinate residuals of the linear calibration curve, $b$ is the slope of the same curve, $y_{0}$ is the specific experimental XIC value from which the concentration $\mathrm{x}_{0}$ is to be determined, $y_{a v}, x_{a v}$ and $x_{i}$ are, respectively, the average values and the actual values of the experimental points of the calibration curve. The letters $m$ and $n$ in the formula respectively stand for the number of repeated readings of $y_{0}$, and the number of calibration points in the regression line.

\section{NMR spectroscopy}

After calibration of the ERETIC peak, the concentration of any compound can be simply measured by the relations (2) and (3) reported by Akoka et al. [1]. 
Table 4

LC-MS calibration curves

\begin{tabular}{lcc}
\hline Standard & Concentration range (pmol of standard compound in the injected sample) & Linear regression \\
\hline Cho & $2-60 \mathrm{pmol}$ & $\mathrm{f}(\mathrm{x})=2916.6717 \mathrm{x}+33105.3858$ \\
& & $\mathrm{R}^{2}=0.9686$ \\
PCho & $20-620 \mathrm{pmol}$ & $\mathrm{f}(\mathrm{x})=1798.7706 \mathrm{x}-19980.8081$ \\
& & $\mathrm{R}^{2}=0.9892$ \\
GPCho & $8-70 \mathrm{pmol}$ & $\mathrm{f}(\mathrm{x})=2676.0714 \mathrm{x}+11325.9524$ \\
& & $\mathrm{R}^{2}=0.9734$ \\
\hline
\end{tabular}

$$
\text { [Comp }]=\mathrm{k} \text { [ERETIC] } \mathrm{A}_{\text {Comp }} / \mathrm{A}_{\text {ERETIC }}
$$

where [Comp] is the concentration of analyte, $\mathrm{k}$ takes into account the number of protons per chemical group, $\mathrm{A}_{\mathrm{Comp}}$ is the area of the peak to be quantified, $\mathrm{A}_{\text {ERETIC }}$ is the area of the ERETIC peak. The value [ERETIC] is the equivalent concentration of the ERETIC line determined after a calibration acquisition by the expression

$$
[\text { ERETIC }]=[\mathrm{REF}] \mathrm{A}_{\text {ERETIC }} / \mathrm{A}_{\mathrm{REF}}
$$

where $[\mathrm{REF}]$ is the concentration of a calibration solution and $A_{R E F}$ is the area of the calibration peak.

To calibrate the ERETIC peak we acquired the ${ }^{1} \mathrm{H}$ spectrum of a reference mixtures of standard Cho, PCho and GPCho, each one $1 \mathrm{mM}$ in $0.5 \mathrm{ml}$ of $\mathrm{D}_{2} \mathrm{O}$. We adopted as equivalent concentration for the artificial peak, the average of the values derived from the three reference compounds. The precision of the obtained average value for [ERETIC], namely $0.028 \mathrm{mM}$, was good as shown by the relative standard deviation, namely 0.00048 .
The analytes quantification was certainly affected by the low intensity of the target peaks compared to noise; the error level in metabolite concentrations was derived by error propagation rules, from the error $\left(\mathrm{S}_{\mathrm{A}}\right)$ associated to each integral area value $\left(\mathrm{A}_{\mathrm{Comp}}\right)$. The $\mathrm{S}_{\mathrm{A}}$ value was calculated from the spectrum signal to noise ratio (SNR) according to the following formula:

$$
\mathrm{S}_{\mathrm{A}}=\frac{\mathrm{A}_{\text {Comp }}}{\mathrm{SNR}}
$$

The SNR value was determined for each 1D spectrum by the software TOPSPIN according to the definition:

$$
\mathrm{SNR}=\frac{\text { maxval }}{2 \cdot \text { noise }}
$$

where maxval is the highest intensity in the signal region and noise factor is referred to a fixed region where no signals are detected. 


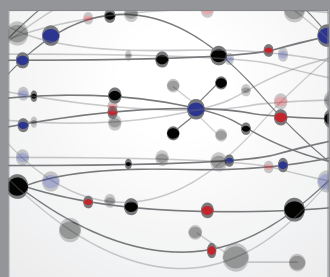

The Scientific World Journal
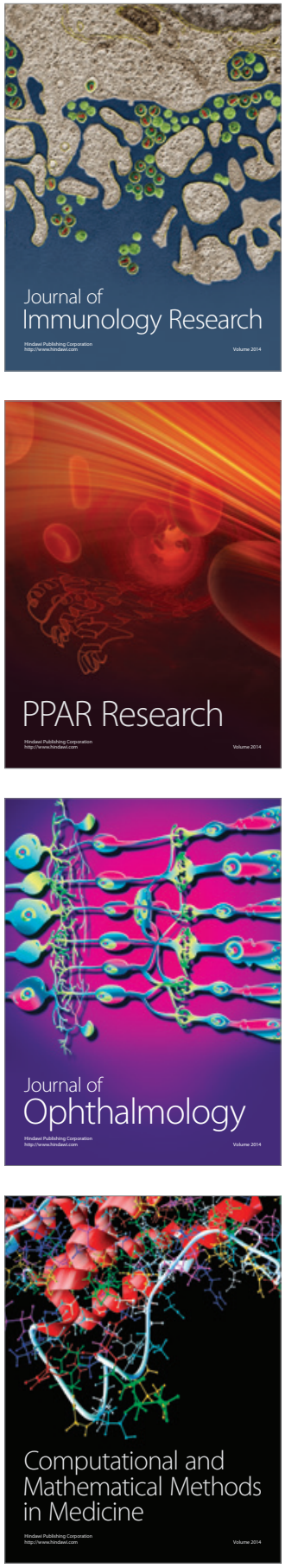

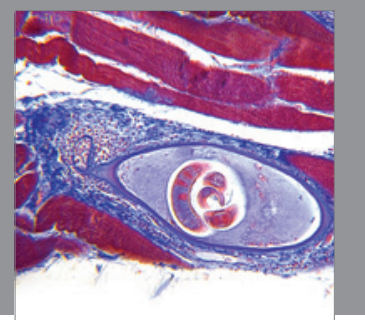

Gastroenterology

Research and Practice
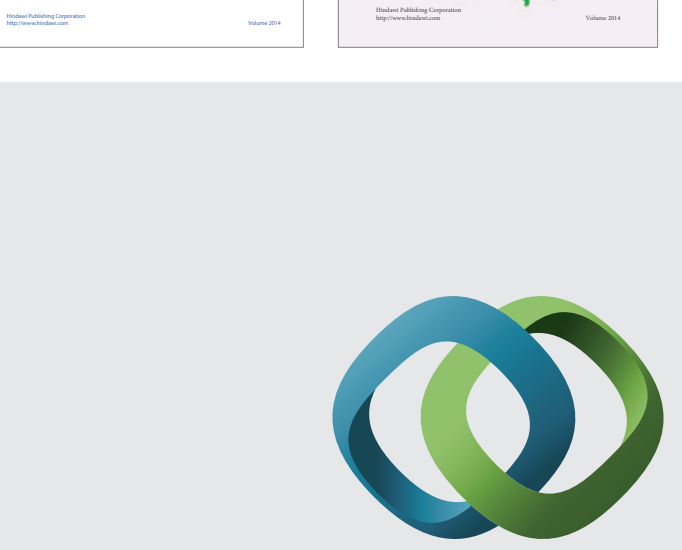

\section{Hindawi}

Submit your manuscripts at

http://www.hindawi.com
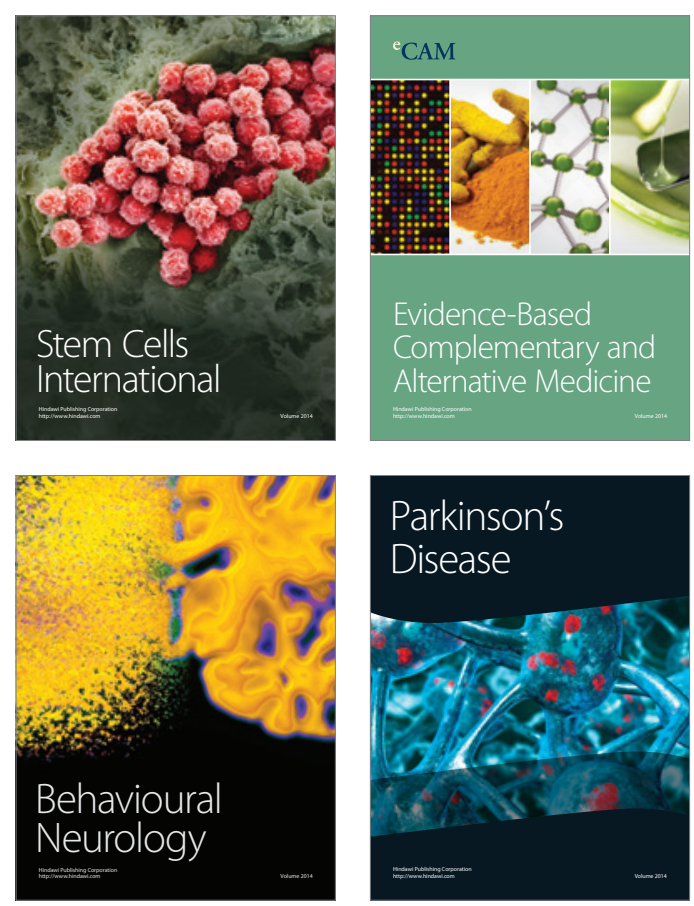

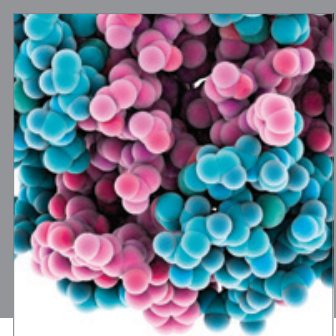

Journal of
Diabetes Research

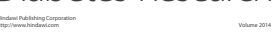

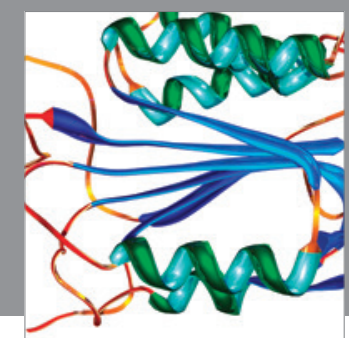

Disease Markers
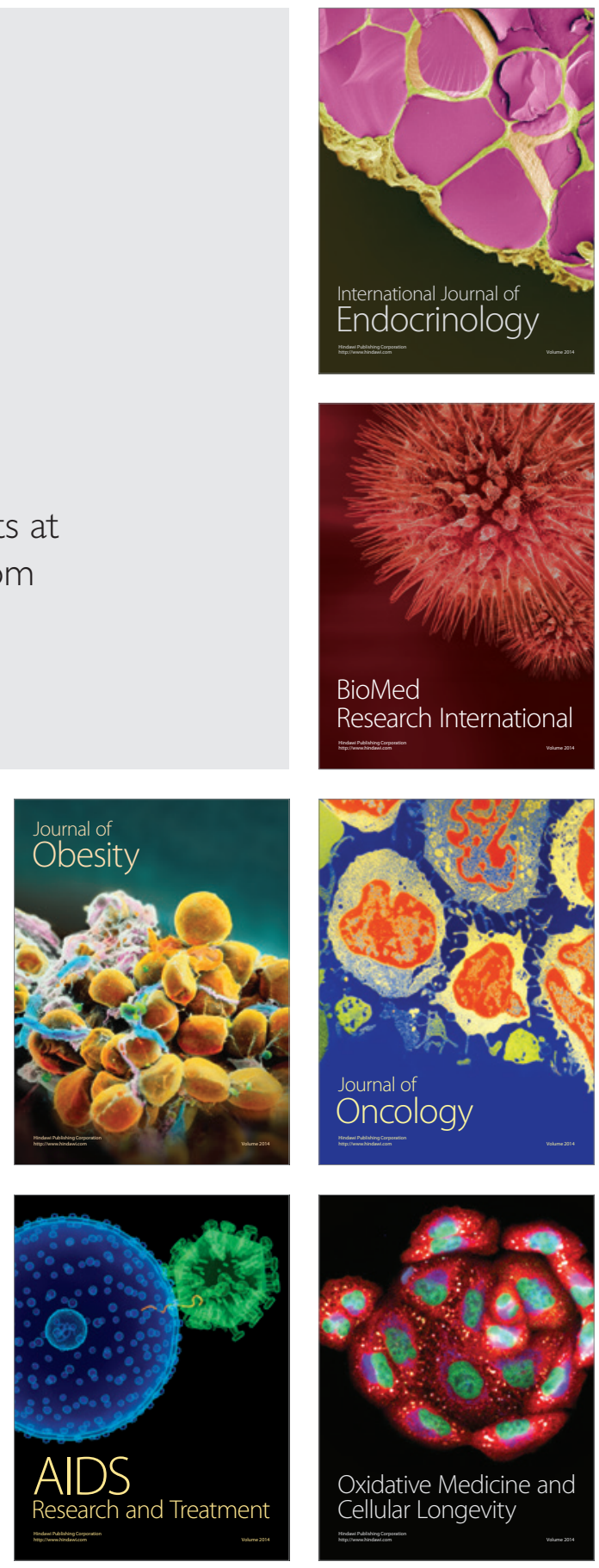